\title{
La educación por competencias como medio para facilitar la toma de control del aprendizaje por el estudiante
}

\author{
Francisco Germain, Consuelo Pérez-Rico
}

Introducción. Clásicamente, el papel de los alumnos ha sido el de elemento pasivo en el marco de una enseñanza monodireccional donde el alumno tenía pocas posibilidades de controlar su aprendizaje y, por tanto, de implicarse activamente en él.

Materiales y métodos. Para fomentar la toma de control del aprendizaje por el alumnado se han diseñado unas estrategias consistentes en la aplicación de una serie de competencias transversales. Se ha elegido como asignatura la fisiopatología del Grado de Fisioterapia del curso 2011-2012 porque su gran proporción de seminarios permite una mayor participación del alumnado. Después, se han seleccionado una serie de competencias: la capacidad de búsqueda de información y evaluación crítica, la capacidad de análisis y síntesis, la capacidad de dar respuesta adecuada a las preguntas, la capacidad crítica sobre lo leído y escuchado plasmada en forma de preguntas y, por último, la claridad y calidad en la exposición oral de un tema previamente propuesto.

Resultados. Con la implantación y desarrollo de las competencias designadas, además de mejorar dichas competencias, las calificaciones del alumnado han mejorado de forma estadísticamente significativa respecto de las de los dos cursos anteriores (test exacto de Fisher). De forma global, en el curso en que se ha implementado la innovación, un 82\% del alumnado superó el curso en la convocatoria ordinaria, respecto al 53\% y el $44 \%$ de los dos cursos anteriores.

Conclusión. Las actividades que se realizan para lograr la adquisición de las competencias transversales fundamentales facilitan a los estudiantes la toma de control de su aprendizaje y mejoran dicho aprendizaje.

Palabras clave. Aprendizaje por competencias. Competencias transversales. Modelos de aprendizaje.

\section{Competency-based education as a way to facilitate the take over of learning by the student}

Introduction. Traditionally, the students' role has been a passive element in the framework of a one-way teaching process, where students had few perspectives of controlling their learning and, therefore, of actively getting involved on it.

Materials and methods. In order to promote the take over of learning by the students, a series of strategies, which consisted on applying a series of transversal competences, were designed. The subject chosen for this purpose was Pathophysiology, belonging to the Degree in Physiotherapy of the academic year 2011/2012, because the relative high amount of seminars in this subject enables a great student participation. Then, a range of competences were selected, such as the ability to search for information and critically evaluate it; the capacity for analysis and synthesis; the capacity to adequately answer questions; the critical thinking ability concerning what has been read and heard, that is translated into the capacity of making questions about the subject; and, finally, the clarity and quality when presenting orally a previously proposed topic.

Results. With the implementation and development of the designated competences, in addition to the enhancement of these skills in the students, their scores improved in a statistically significant manner with respect to those from the two previous years (Fisher's exact test). In the academic year which this innovation was implemented, $82 \%$ of the students passed the course in the ordinary call, as compared to $53 \%$ and $44 \%$ of the two previous years.

Conclusion. The activities carried out to achieve the acquisition of key transversal competences have facilitated the students to take over control of their own learning and, importantly, to make it more efficient.

Key words. Competency-based learning. Learning models. Transversal competence.

Departamento de Fisiología; Universidad de Alcalá (F. Germain). Hospital Príncipe de Asturias (C. Pérez-Rico). Alcalá de Henares, Madrid, España.

Correspondencia: Prof. Francisco Germain. Departamento de Fisiología. Universidad de Alcalá. Campus Universitario. A-2, km 31. E-28871 Alcalá de Henares (Madrid).

E-mail:

francisco.germain@uah.es

Agradecimientos:

Dra. Margarita Barón, profesora emérita de la Universidad de Alcalá, por la revisión del manuscrito y por las orientaciones e ideas que surgieron durante su planificación. Dr. Pedro de la Villa, catedrático de la Universidad de Alcalá, por las facilidades y apoyo recibidos para poner en marcha esta experiencia educativa y por los comentarios sobre algunas de las actividades. Dra. Gómez-Vicente, por la revisión del inglés.

Conflicto de intereses: No declarado.

Competing interests: None declared.

(c) 2014 FEM 


\section{Introducción}

Tras la declaración de Bolonia (1999), en el año 2000 se propuso la 'estrategia de Lisboa' como respuesta a los múltiples retos que emanan de la globalización. Se genera el concepto de 'espacio europeo', asentado en tres pilares: la educación, la investigación y la innovación (triángulo del conocimiento). Se trata de un cambio de paradigma en la educación superior: pasar de la enseñanza al aprendizaje centrando todo el proceso en un estudiante activo y participativo teniendo especialmente en cuenta la formación en competencias, es decir, la adquisición de habilidades, actitudes y aptitudes basadas en la amplitud del conocimiento previo. En educación médica, Miller había definido en 1990 la pirámide de competencias clínicas [1]. Posteriormente, en 1996, la Brown University School of Medicine inauguró un nuevo plan de estudios basado en competencias transversales. El currículo se construía sobre una base de conocimientos, nueve competencias que el estudiante debía desarrollar y la valoración del desempeño del estudiante.

Las competencias son transversales y específicas. Las primeras son las comunes a diferentes disciplinas: capacidad de comunicación, de trabajo en equipo, de liderar y ser liderado, de integración en un ambiente multidisciplinar, capacidad para el autoaprendizaje y, por tanto, para el aprendizaje permanente, para el desarrollo profesional y la responsabilidad social, etc. Respecto de las competencias específicas, son aquellas que se adquirirán en función de la profesión concreta que se estudie [2].

Dichas competencias deben evaluarse durante el proceso de aprendizaje. El sistema de evaluación basado en competencias promueve hábitos de autorregulación y prácticas reflexivas [3,4]. De hecho, el objetivo principal de esta retroalimentación es permitir al estudiante monitorizar la adquisición de las competencias y planificar los cambios necesarios para su consecución [5].

Continuando con el ejemplo de la educación en ciencias de la salud, con las recientes reformas educativas se ha pasado de formación médica continuada a desarrollo profesional continuo, en que el profesional lidera intencionadamente, en su afán de servicio a la sociedad, la actualización de sus conocimientos, habilidades y competencias para, modificando su conducta y su práctica en el ejercicio profesional, dar respuesta a la evolución científica, tecnológica y social [2]. Para que este cambio de mentalidad se instale de forma más profunda la orientación adecuada debe comenzar ya en el estudiante de grado.
Un aspecto que no suele considerarse en la bibliografía es la opinión de quien aprende [6]. Sin embargo, cada vez hay más evidencias que sugieren la importancia de las experiencias subjetivas de quien aprende y su valoración de éstas, en la aceptación de la retroalimentación formativa [5]. Por todo ello, profesores y estudiantes deben afrontar la docencia con el mismo espíritu: aprender y revisar constantemente sus conocimientos. Sólo fruto del análisis y la renovación del conocimiento puede éste avanzar.

Siguiendo esta concepción, en el camino del aprendizaje resulta necesario que el alumno tome conciencia previa del motivo por el cual quiere aprender. Una vez que se conoce y acepta dicha motivación, es fundamental desarrollar las actitudes que le permitan mejorar. En este sentido, la evaluación ayuda de forma significativa a que adquiere la responsabilidad de su propio aprendizaje, especialmente en la educación basada en competencias [7-9].

El objetivo principal de la educación sanitaria de pregrado es enseñar las habilidades necesarias para el razonamiento clínico [10]. Sin embargo, nuestra comprensión de cómo se produce la toma de decisiones y de cómo se adquieren esas habilidades aún no se conoce por completo [11-13]. Con la intención de ayudar a adquirir esas habilidades que faciliten la toma de control del aprendizaje, se ha hecho hincapié en una serie de competencias: la capacidad de búsqueda de información con una evaluación crítica de su importancia, la capacidad de análisis y síntesis, la capacidad de dar respuesta adecuada a las preguntas que se formulen, la capacidad crítica y la de hacer las preguntas que correspondan y, por último, la claridad y calidad en la exposición oral.

Con la intención de luchar contra la tradicional pasividad del alumno, se ha aplicado un cambio metodológico. Éste pretende fomentar la participación activa del alumno con la intención de estimular la construcción personal de un conocimiento significativo y relevante, en la que los contenidos de la asignatura se reactiven de forma constante mediante la formación de interrelaciones entre ellos. La asignatura elegida para aplicar este nuevo enfoque metodológico ha sido la fisiopatología dentro del Grado de Fisioterapia.

El objetivo fundamental de la innovación aplicada ha consistido en corregir la pasividad del alumnado. El medio utilizado para ello ha sido la promoción de un aprendizaje activo mediante la adquisición y desarrollo de una serie de competencias transversales. 


\section{Materiales y métodos}

\section{Planificación didáctica}

Para el diseño de la planificación didáctica, adaptamos la seguida por Gimeno-Sacristán y Pérez-Gómez [14]. El esquema incluye la selección de competencias, la elección de los contenidos, el diseño de estrategias y, finalmente, la evaluación de todo lo anterior.

Tras implementar la innovación, los resultados se han analizado y discutido con observadores externos y con los propios alumnos para evaluar la percepción de logro de los objetivos y analizar las incidencias que podrían haber surgido en su desarrollo.

El objetivo fundamental que se plantea con esta innovación es corregir la pasividad del alumno, probablemente inducida por la metodología docente tradicional. Para ello se ha desarrollado un plan basado en competencias transversales que fomenten la toma de control del aprendizaje. Dichas competencias se han utilizado como instrumento para alcanzar los objetivos cognitivos en los diferentes módulos. Las competencias, sus criterios y procedimientos de evaluación se muestran en la tabla I.

Los contenidos se han seleccionado, organizado y secuenciado en función de su relevancia, representatividad y capacidad potenciadora del aprendizaje. Los límites vienen marcados por la guía docente en la cual se desarrolla la formación académica en el marco del plan de estudios, se elabora el perfil profesional, el tiempo del que se dispone y los recursos con los que se cuenta.

Una vez decididas las competencias y los contenidos, se diseñan las estrategias metodológicas para conseguir que esos alumnos, en ese momento y con esos recursos y condiciones, 'emprendan' el camino de 'aprender a aprender'. De gran trascendencia ha sido determinar cuál ha de ser el papel del profesor. Desde un principio se ha querido desplazar el protagonismo del profesor al alumno; para ello el profesor ha debido mantenerse aparte, como un observador experto que sólo interviene si es necesario y no para ser el centro de atención.

\section{Descripción de la población}

La innovación que se presenta ha sido aplicada en un curso de 79 estudiantes de fisiopatología del Grado de Fisioterapia de la Universidad de Alcalá durante el curso académico 2011-2012; la edad media de estos estudiantes es de 18 años; la proporción mujer/hombre, de 1,47; la forma de acceso a estos estudios, mayoritariamente vía pruebas de ac-
Tabla I. Criterios y procedimientos de evaluación de las competencias.

\begin{tabular}{lcc}
\hline $\begin{array}{l}\text { Búsqueda de información } \\
\text { y evaluación crítica }\end{array}$ & $\begin{array}{c}\text { Información básica expuesta } \\
\text { y su ordenación jerárquica }\end{array}$ & Presentación oral y defensa \\
\hline $\begin{array}{l}\text { Análisis y síntesis de los } \\
\text { materiales bibliográficos }\end{array}$ & $\begin{array}{c}\text { Grado de profundidad } \\
\text { alcanzado en el análisis }\end{array}$ & $\begin{array}{c}\text { Preguntas orales y escritas. } \\
\text { Respuestas dadas a las } \\
\text { preguntas orales }\end{array}$ \\
\hline $\begin{array}{l}\text { Dar respuesta adecuada a las } \\
\text { preguntas que se les formule }\end{array}$ & Idoneidad de \\
\hline $\begin{array}{l}\text { Cuestionarse lo que lee o } \\
\text { escucha y hacer preguntas }\end{array}$ & $\begin{array}{c}\text { lalidad de las preguntas orales } \\
\text { y de las escritas enviadas }\end{array}$ & $\begin{array}{c}\text { Respuestas dadas a las } \\
\text { preguntas tras la exposición }\end{array}$ \\
\hline $\begin{array}{l}\text { Claridad y calidad } \\
\text { en la exposición oral }\end{array}$ & $\begin{array}{c}\text { Claridad, corrección } \\
\text { y cuidado expositivo }\end{array}$ & Presentación oral \\
\hline
\end{tabular}

ceso a la universidad (selectividad); la nota máxima de acceso, de 12,133, y la mínima, de 5,24, correspondiente a un alumno de élite deportiva.

Dicha asignatura se cursa en el segundo cuatrimestre del primer año de la titulación debido a que se necesitan unos conocimientos básicos que se imparten previamente en fisiología en el primer cuatrimestre.

La metodología propuesta para alcanzar esas competencias se aplica en los seminarios, ya que en ellos surge una implicación mucho más activa por parte del alumno. El contenido de la asignatura se organiza en módulos temáticos; cada módulo consta del mismo número de temas que de seminarios. Sin embargo, mientras que la audiencia en los temas es la totalidad del alumnado durante una hora, en los seminarios el número de alumnos es reducido (un tercio) y su duración es de dos horas (es decir, representan el 66\% de la actividad presencial del alumnado).

\section{Estrategia metodológica}

La guía docente publicada en la web de la Universidad de Alcalá refleja ampliamente los objetivos y contenidos del programa, por lo que en el primer encuentro con los alumnos se explica única y exhaustivamente la innovación, especialmente lo referido a las competencias (Tabla I) y la forma de evaluarlas (Fig. 1).

Una semana antes de cada seminario, el profesor expone en la plataforma virtual diez preguntas que pretenden recoger los contenidos básicos del tema tratado en ese seminario, así como el material bi- 
Figura 1. Plantilla para la evaluación de los alumnos.

\begin{tabular}{|l|l|l|l|l|l|}
\hline NOMBRE DEL ALUMNO: & $\begin{array}{c}1 \\
\text { No } \\
\text { adecuado }\end{array}$ & 2 & 3 & $\begin{array}{c}4 \\
\text { Muy } \\
\text { Suficiente }\end{array}$ & $\begin{array}{c}\text { Adecuado } \\
\text { adecuado }\end{array}$ \\
\hline \begin{tabular}{l|l|l|l|l|}
\hline CONTENIDO & & & \\
\hline $\begin{array}{l}\text { Estructura, orden y coherencia } \\
\text { de la información que se presenta }\end{array}$ & & & & \\
\hline $\begin{array}{l}\text { Actualidad de la información } \\
\text { (cuando pueda constatarse) }\end{array}$ & & & & \\
\hline $\begin{array}{l}\text { Presentación de los puntos } \\
\text { importantes del tema }\end{array}$ & & & & \\
\hline $\begin{array}{l}\text { Capacidad de resumen } \\
\text { o sintesis final }\end{array}$ & & & & \\
\hline $\begin{array}{l}\text { Respuestas dadas a las } \\
\text { preguntas formuladas }\end{array}$ & & & & \\
\hline
\end{tabular}
\end{tabular}

FORMA:

\begin{tabular}{|c|l|l|l|l|}
\hline $\begin{array}{l}\text { El vocabulario técnico utilizado es } \\
\text { el apropiado al tema que se trata }\end{array}$ & & & & \\
\hline No se usan muletillas verbales & & & & \\
\hline Cambios de tono & & & & \\
\hline Gesticulación & & & & \\
\hline Seguridad y convioción & & & & \\
\hline $\begin{array}{l}\text { Presentación visual ágil y atractiva, } \\
\text { sin abuso del powerpoint }\end{array}$ & & & & \\
\hline
\end{tabular}

PREGUNTAS AL PONENTE

RELEVANCIA DE LA PREGUNTA

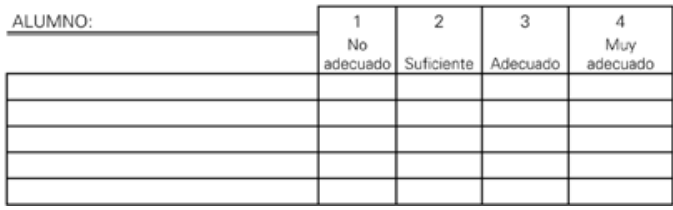

bliográfico necesario para responderlo. Dichas preguntas están orientadas a pedir las causas y los procesos que llevan a determinada patología (p. ej., 'causas y mecanismos patogénicos del hipertiroidismo'), presentar problemas en que se 'justifique porque un hipertiroidismo primario puede producir una hiperprolactinemia, incidir en diferencias críticas entre patologías que pudieran confundirse ('diferenciación de las acidosis y alcalosis según sean de causa metabólica o respiratoria) o hacer preguntas que para contestarlas tengan que relacionar con temas previos ('¿por qué el insomnio familiar letal encaja en las enfermedades priónicas?').

Las respuestas a cada una de las preguntas deben hacerse en formato de presentación oral estructurada atendiendo a las siguientes premisas:

- Todos los alumnos deben tener preparada individualmente una contestación a cada una de las preguntas propuestas, pero sólo uno expondrá de forma pública una respuesta completa. Se les permite que sean ellos los que decidan quién hará las exposiciones en respuesta a las pregun- tas. Para evitar que los alumnos sólo se prepararen aquella pregunta que van a exponer, tras cada exposición se inicia un debate en el que el profesor preguntará y evaluará a varios de los asistentes en relación con el tema tratado en la exposición. La intención es que, al final de cada seminario, todos hayan contestado a cuestiones relacionadas con el tema tratado en dicho seminario. El alumno debe saber que para poder ser calificado, al final del cuatrimestre debe haber hecho al menos tres exposiciones.

- El profesor se abstendrá de hacer una exposición paralela tras la de cada estudiante, con el objetivo de no rebajar el interés de los alumnos para con el ponente. Tras el seminario, el profesor sí puede exponer la presentación en la plataforma virtual, explicando que sólo es complementaria de las presentaciones de los alumnos.

- Por último, se insiste en la importancia de una correcta exposición pública, debiendo cuidar no sólo la ordenación y estructuración de ideas entorno a un objetivo, sino también lo referente al correcto uso de los medios audiovisuales, la fluidez, dicción, gesticulación y capacidad de expresión. Con ello se intenta generar una seguridad en el ponente que facilite su razonamiento a la hora de contestar las preguntas que se le formulen.

Con la intención de combatir la pasividad de los alumnos que escuchan al ponente, se insta a que sean ellos quienes planteen preguntas al finalizar la exposición. Dichas preguntas deben demostrar que se han preparado el tema y que han estado atentos a la exposición, lo que se incentiva con una mejora en la nota. Por tanto, para poder responder a las preguntas, el ponente debe tener cierta seguridad de conocer el tema con la profundidad necesaria, más allá de lo simplemente expuesto. Cuando ningún alumno tenga nada más que aportar, el profesor pedirá que el alumno-ponente haga una síntesis que sirva de cierre.

Terminadas las presentaciones orales, se proyectan en la pantalla una serie de preguntas de respuesta múltiple (test) en relación al tema tratado y a las que deben contestar en papel eligiendo la respuesta correcta. La intención de este ejercicio es que sirva de repaso y síntesis de los conceptos importantes enfocando su atención en aspectos concretos. Una vez recogidos los ejercicios, se proyectan las respuestas correctas y se debate sobre ellas.

Independientemente de las actividades anteriores, se pide cada semana al alumnado que confeccione una pregunta tipo test. Las preguntas deben remitirse al profesor con la respuesta correcta mar- 
Tabla II. Grado de satisfacción del alumnado: resultados obtenidos.

\begin{tabular}{|c|c|c|c|c|c|c|c|}
\hline & & $\begin{array}{l}\text { Nada } \\
\text { (1) }\end{array}$ & $\begin{array}{l}\text { Algo } \\
\text { (2) }\end{array}$ & $\begin{array}{l}\text { Suficiente } \\
\text { (3) }\end{array}$ & $\begin{array}{l}\text { Bastante } \\
\text { (4) }\end{array}$ & $\begin{array}{l}\text { Todo } \\
(5)\end{array}$ & $\begin{array}{l}\text { Escala } \\
\text { Likert }\end{array}$ \\
\hline \multirow{11}{*}{$\begin{array}{l}\text { Seminarios: } \\
\text { presentación oral }\end{array}$} & Búsqueda bibliográfica y preparación de material & 1 & 6 & 17 & 32 & 3 & 3,51 \\
\hline & Ordenación jerárquica de las ideas & 1 & 8 & 20 & 27 & 3 & 3,39 \\
\hline & Confección del trabajo & & 10 & 24 & 25 & & 3,25 \\
\hline & Exposición de mi presentación & & 7 & 17 & 33 & 2 & 3,51 \\
\hline & Responder a las preguntas que me puedan formular & 2 & 14 & 24 & 15 & 3 & 3,05 \\
\hline & Hacer preguntas al que expone & 1 & 21 & 27 & 9 & 1 & 2,80 \\
\hline & Vencer el miedo escénico & 4 & 20 & 14 & 16 & 5 & 2,97 \\
\hline & Expresarme mejor en público & & 17 & 17 & 22 & 3 & 3,19 \\
\hline & Ordenar y estructurar jerárquicamente el trabajo & 1 & 3 & 27 & 25 & 4 & 3,47 \\
\hline & Prepararme más allá de lo que expongo & 1 & 16 & 21 & 17 & 3 & 3,09 \\
\hline & Contestar con base científica & 3 & 12 & 19 & 24 & & 3,10 \\
\hline \multirow{9}{*}{ Preguntas del test } & Las elaboradas por mí me han ayudado a: & & & & & & \\
\hline & Buscar los datos clave de forma selectiva & 1 & 7 & 22 & 28 & 1 & 3,36 \\
\hline & Reforzar el recuerdo de dichos datos & 3 & 7 & 15 & 28 & 6 & 3,46 \\
\hline & Interrelacionar esos datos con otros del tema & 2 & 16 & 23 & 15 & 3 & 3,02 \\
\hline & Favorecer la interacción científica con compañeros & 6 & 19 & 25 & 8 & 1 & 2,64 \\
\hline & Las proyectadas en los seminarios me han ayudado a: & & & & & & \\
\hline & Orientar el modo de estudio & 2 & 6 & 23 & 25 & 2 & 3,33 \\
\hline & Controlar el tiempo de respuesta & 3 & 13 & 18 & 18 & 6 & 3,19 \\
\hline & Priorizar en caso de penalización de la respuesta & 4 & 7 & 23 & 20 & 4 & 3,22 \\
\hline
\end{tabular}

cada y deben incluir un razonamiento explícito sobre la selección de la respuesta supuestamente correcta. De esta forma, el profesor puede hacer un seguimiento del progreso en el aprendizaje del alumnado y también de la atención que presta a los datos relevantes.

Al término del cuatrimestre se entrega al alumnado un cuestionario para que evalúen su percepción de mejora en diversos aspectos que contienen cada una de las competencias transversales de la tabla I. Dicho cuestionario se muestra con los resultados obtenidos en el tabla II.
Los resultados se analizaron estadísticamente con el programa GraphPad InStat v. 5.00 para Windows.

\section{Resultados}

Con el transcurso de los seminarios, cada alumno mostró una mejora importante en las diferentes competencias trabajadas, tanto de aquellas que utilizaron las exposiciones y debates posteriores como las que se valieron de la elaboración de preguntas de test, mostrando una mayor precisión y relevancia. 
Figura 2. Grado de satisfacción del alumnado en la preparación de la presentación oral y exposición. Encima de cada columna se muestra el porcentaje de alumnos correspondiente. En abscisas se muestra la escala de Likert de 5 puntos (nada: 1; algo: 2; suficiente: 3; bastante: 4; todo: 5), y en ordenadas, el número de alumnos que eligieron esa opción.
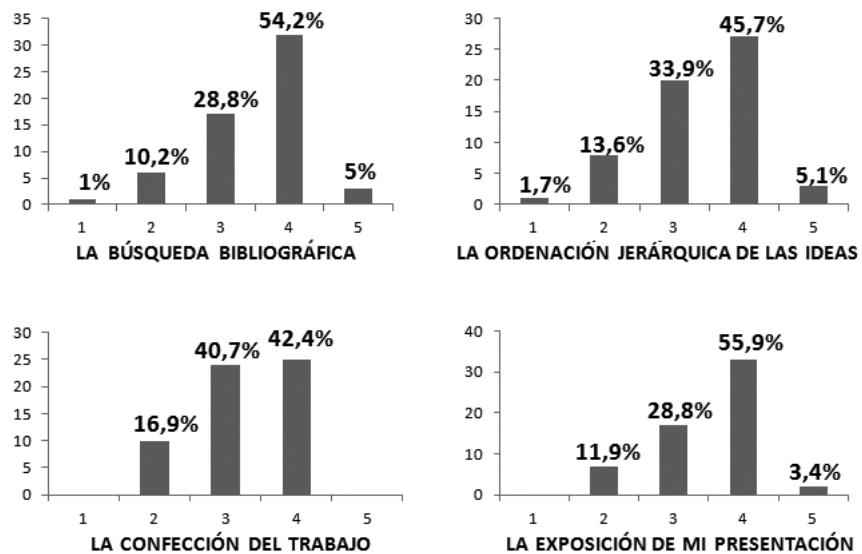

Figura 3. Grado de satisfacción del alumnado en la preparación para el debate (a) y los aspectos emocionales de las presentaciones (b). Encima de cada columna se muestra el porcentaje de alumnos correspondiente. En abscisas se muestra la escala de Likert de 5 puntos (nada: 1; algo: 2; suficiente: 3; bastante: 4; todo: 5), y en ordenadas, el número de alumnos que eligieron esa opción.
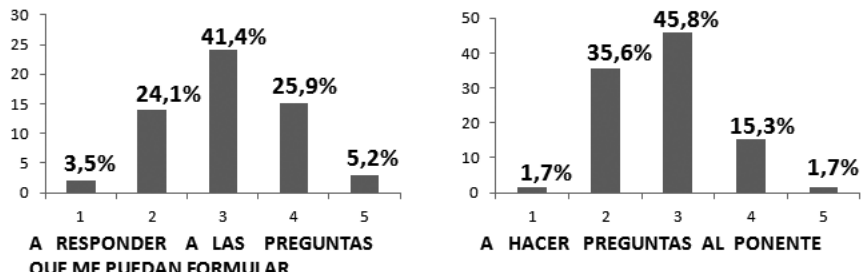

A HACER PREguntas AL PONENTE QUE ME PUEDAN FORMULAR
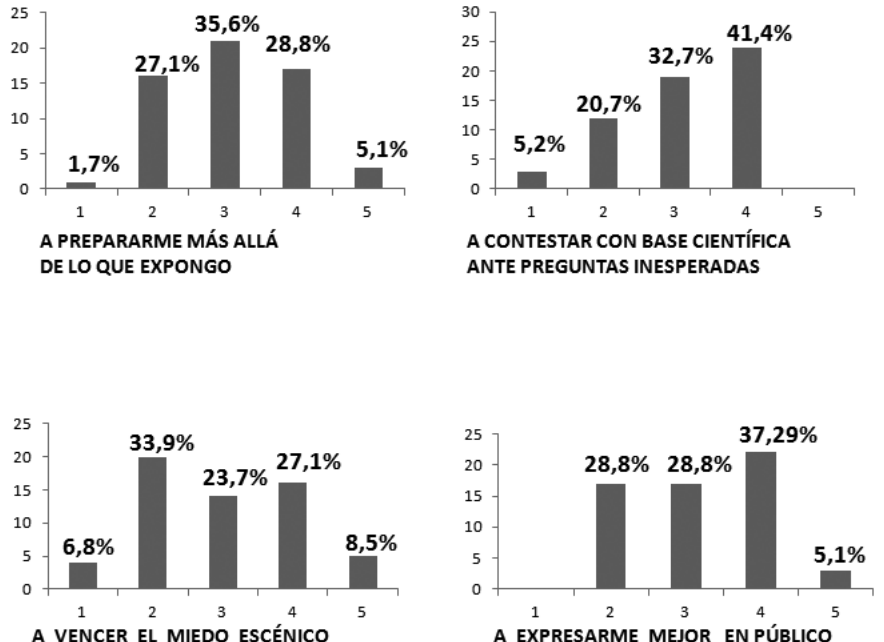

La percepción de los alumnos respecto a distintos aspectos relacionados con la adquisición de las competencias deseadas se ha recogido en los cuestionarios (Tabla II). En dicho cuestionario se aplica la escala de Likert de 5 puntos (nada: 1; algo: 2; suficiente: 3 ; bastante: 4 ; todo: 5 ).

\section{Respuestas al cuestionario (Tabla II)}

El cuestionario cumplimentado por los alumnos muestra que, en general, éstos reconocen como positiva su tarea en los seminarios, siendo la media ponderada de sus respuestas superior a 'suficiente' (más de 3 en la escala de Likert).

En los aspectos relativos a la preparación de la presentación oral y su exposición, los porcentajes más elevados corresponden a la respuesta 'bastante' (4 en la escala de Likert) (Fig. 2). Respecto a la preparación para el debate, las respuestas se desplazan hacia un menor logro tanto en la capacidad de responder a las preguntas como en la de hacer preguntas al ponente.

La percepción de una preparación profunda que permite responder a posibles preguntas muestra una distribución tipo 'curva de Gauss'. Dentro de este apartado, sólo la capacidad de responder con base científica a las preguntas formuladas muestra una valoración más favorable para la respuesta 'bastante' (Fig. 3a).

Respecto a aspectos emocionales de las presentaciones, como el miedo escénico y la expresión pública, tan culpables de inhibir el aprendizaje al dificultar el libre diálogo público, las opiniones se distribuyen casi a partes iguales entre 'poco', 'suficiente' y 'bastante' (Fig. 3b).

Respecto a las preguntas tipo test proyectadas al final del seminario, los alumnos estiman que les ayudan significativamente a orientar el modo de estudio. Igualmente, parecen ayudarles a priorizar las respuestas ante la posibilidad de que puedan ser penalizadas (Fig. 4a). Así mismo, la elaboración de preguntas de respuesta múltiple por el propio alumno muestra una percepción importante de mejora en la capacidad de buscar datos clave de forma selectiva y de reforzar el recuerdo de dichos datos. El pico de respuesta porcentual para ambos se encuentra en la respuesta 'bastante' (Fig. 4b). Dentro de la confección de las preguntas de test, existen otros aspectos cuya valoración por parte de los alumnos no es tan positiva: el fomento de la interrelación de datos y la estimulación de la interacción científica con los compañeros mediante el intercambio de preguntas y conocimientos concretos. 
Comparación de los resultados académicos

de esta asignatura con los obtenidos

en los dos cursos anteriores

En la Universidad de Alcalá, la asignatura de fisiopatología en el Grado de Fisioterapia, en el marco de los nuevos planes de estudio orientados hacia el Espacio Europeo de Educación Superior, se está impartiendo desde el curso 2009-2010; en la actualidad son ya tres años de experiencia. En los dos cursos precedentes, el desarrollo de la asignatura ha resultado similar al aplicado en el curso 2011-2012. Sin embargo, en los dos cursos previos, la estrategia docente en los seminarios no incluía la práctica de competencias transversales; simplemente se realizaban preguntas a un alumnado seleccionado aleatoriamente.

En el curso 2011-2012 se ha presupuesto que ciertas competencias básicas -o transversales- son necesarias para combatir la pasividad del alumno en su aprendizaje, razón por la cual el esquema metodológico diseñado se explica exhaustivamente a los estudiantes con el objetivo de conseguir su máxima implicación y colaboración. Por tanto, se llamará 'curso de la innovación' -objeto de este estudioal 2011-2012.

Las competencias (Tabla I) han mejorado a lo largo del curso. Sin embargo, lo más relevante del estudio es que los resultados de las pruebas finales correspondientes al examen ordinario demuestran que la adquisición de conocimientos también ha mejorado. En la figura 5 se muestran las calificaciones comparadas entre estos tres cursos del grado. El número de no presentados disminuye en el curso de la innovación, pero la disminución más significativa se encuentra en el número de suspensos, que pasa del $31 \%$ y $36,6 \%$ en los dos cursos anteriores a un 7,6\% en 2011-2012. Del mismo modo, el número de alumnos calificados con aprobado aumenta algo más de un 30\% en el curso de la innovación, al tiempo que las calificaciones más elevadas también sufren un importante incremento (Fig. 5).

Para el análisis estadístico se ha aplicado el test exacto de Fisher, comparando los cursos por parejas. En la primera tabla de contingencia se introducen dos grupos, el curso 2009-2010 contra el curso de la innovación, y dos categorías, curso superado (conjunto de aprobados, notables, sobresalientes y matrículas de honor) y curso no superado (suspensos y no presentados). Dicho análisis muestra una diferencia estadísticamente significativa $(p<0,0001)$. Igualmente, la comparación entre el curso 2010-2011 y el curso de la innovación muestra una diferencia estadísticamente significativa $(p<0,0001)$. Sin em-
Figura 4. Grado de satisfacción del alumnado con las preguntas proyectadas (a) y con las elaboradas (b). Encima de cada columna se muestra el porcentaje de alumnos correspondiente. En abscisas se muestra la escala de Likert de 5 puntos (nada: 1; algo: 2; suficiente: 3; bastante: 4; todo: 5), y en ordenadas, el número de alumnos que eligieron esa opción.

a
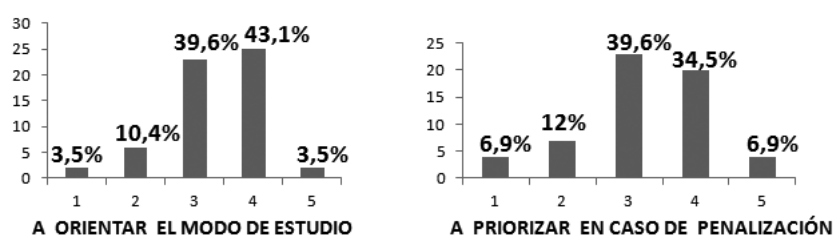

DE LA RESPUESTA

b
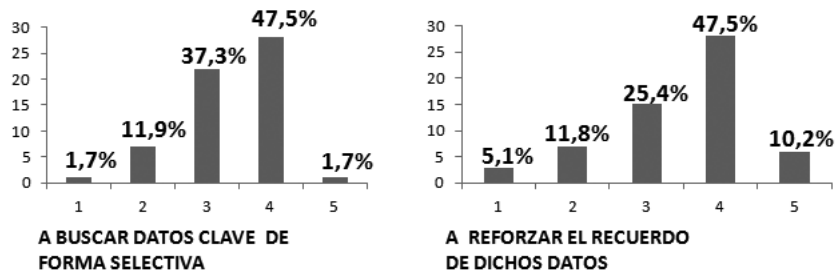

bargo, no hay diferencias significativas en la comparación entre los cursos 2009-2010 y 2010-2011.

En otras palabras, la mejora de las competencias ha propiciado una mejora significativa del conocimiento de la asignatura. Es una experiencia docente realizada en sólo un curso académico. Para tener resultados concluyentes es preciso disponer de datos relativos a más cursos. Sin embargo, también es cierto que se han comparado muestras de población homogéneas en número, edad y procedencia académica de la población; por otra parte, los resultados comparados son de la misma asignatura e idénticos contenido y desarrollo de tiempo.

\section{Discusión}

Las tendencias modernas en educación apuntan hacia un modelo basado en resultados (outcomes), en el cual se hace énfasis en las trayectorias de aprendizaje individual y autodirigido, en la valoración y la retroalimentación de la información, así como en las prácticas reflexivas $[8,15]$. La estrategia docente seguida en el curso de la innovación, a diferencia de los cursos anteriores, estuvo dirigida hacia la toma de control del aprendizaje por parte de los alumnos. 
Figura 5. Comparativa de notas en el curso de la innovación y los dos anteriores. En la figura superior se muestran las curvas de los cursos 2009-2010, 2010-2011 y 2011-2012 (innovación). En abscisas se representan las distintas calificaciones e incluso los no presentados, y en ordenadas, el porcentaje de alumnos que obtuvieron una determinada calificación para cada curso. En las columnas inferiores se representan numéricamente dichos porcentajes. ${ }^{a}$ Diferencia estadísticamente significativa hallada mediante el test exacto de Fisher.

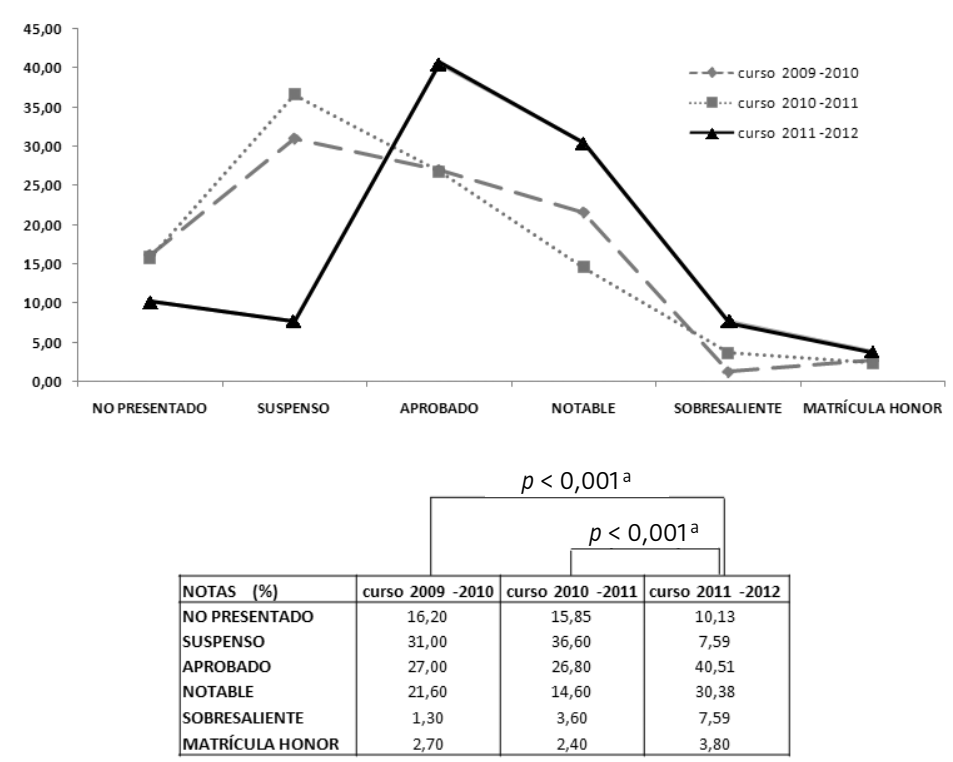

Las poblaciones de estudio en estos tres cursos consecutivos han sido homogéneas en cuanto a procedencia de estudios, proporción de género y edad media. La nota de acceso se encontró entre 7,50 y 8,64 sobre 10 en el curso 2009-2010; entre 10,21 y 12,66 sobre 14 en el curso 2010-2011, y entre 5,24 y 12,133 sobre 14 en el curso 2011-2012. La disminución del límite inferior $(5,24)$ se debe a un pequeño cupo para deportistas de alto rendimiento.

Ha sido de gran importancia contextualizar las competencias con la situación concreta de los alumnos. Se pretende que el alumnado tenga un buen conocimiento tanto de las competencias como de los criterios de calificación que se van a seguir. Por su parte, el profesor debe plantearse su propia actividad en la planificación y diseño de estrategia para el aprendizaje [16]. La relación profesor-estudiante debe guardar un equilibrio entre la cercanía necesaria para proveer de confianza y tutela y la distancia para impedir que se establezcan relaciones académicas o psicológicas perjudiciales [17].

El grado de satisfacción del alumnado tras la realización de las actividades fue, en general, muy positivo. Las presentaciones se hicieron progresiva- mente más precisas y organizadas. Sin embargo, la utilización de recursos como la animación, que las dotaba de mayor brillantez, empezó a poner de manifiesto el riesgo de emplear más esfuerzo en lo formal que en el contenido.

La menor percepción de logro con algunas de las actividades podría haberse originado en su novedad y en la duración del curso, que no permitió una práctica suficiente. En este sentido, algunos alumnos reconocieron un efecto beneficioso y pidieron que se generalizasen a otras asignaturas.

El modelo de libertad seguido en las presentaciones orales responde a la necesidad de disminuir el estrés que supone una actuación pública. Aunque existen pocos estudios que valoren la incidencia y repercusión del estrés en el aprendizaje [18], no hay dudas de la relación existente entre ambos. Aprender a manejarlo se convierte de este modo en un paso necesario para facilitar dicho aprendizaje.

La organización de debates y discusiones facilita el intercambio de conocimientos y el uso de vocabulario profesional. Un lenguaje compartido es una poderosa herramienta que permite asimilar las disciplinas tratadas y adquirir los objetivos del conocimiento profesional necesarios [19]. Por otro lado, las discusiones muestran la importancia de las habilidades comunicativas en el proceso de aprendizaje [20].

La elaboración de las preguntas de test por los alumnos cuenta con una buena aceptación. Para potenciar su valor formativo, el profesor debe corregirlas y enviar dichas contestaciones al alumno tempranamente para que disponga de una retroalimentación de su iniciativa en el tema estudiado. Por su parte, las preguntas proyectadas al final de los seminarios han mostrado su validez como autoevaluación (Fig. 4).

En relación con la evaluación, resulta importante añadir la evaluación por los compañeros con la intención de aumentar los puntos de vista del estudiante y para mejorar su aprendizaje. Parece que la evaluación por los compañeros resulta la más informativa a la hora de evaluar las habilidades personales [21,22]. En general, los estudiantes prefieren ser comparados con sus compañeros de clase, tanto con los que lo hacen mejor, para aprender de ellos, como con los que lo hacen peor, para reforzar su posición [22,23].

En este estudio se ha observado una mejora en el aprendizaje de los contenidos mediante el desarrollo de una serie de actividades nuevas que implicaba la adquisición de ciertas competencias transversales. Para que esto pueda cumplirse es importante que dichas competencias se expliquen claramente a los alumnos y éstos conozcan por qué hacen lo que 
se les pide. Sin embargo, es importante dejar libertad para el ensayo y la equivocación, ya que de la reflexión sobre los errores será de donde más enseñanza obtendrán. En otras palabras, es preciso crear las condiciones para que sea el alumno quien haga suyo el aprendizaje y sea él quien construya su conocimiento, ganando de esta manera en profundidad, impacto y duración.

\section{Bibliografía}

1. Miller GE. The assessment of clinical skills/competence/ performance. Acad Med 1990; 65 (Suppl 9): S63-7.

2. Barón M. El espacio europeo de educación superior (EEES). DPM 2009; 2: 5-14.

3. Dannefer EF, Henson LC. The portfolio approach to competency based assessment the Cleveland Clinic Lerner College of Medicine. Acad Med 2007; 82: 493-502.

4. Fishleder A, Henson LC, Hull A. Cleveland Clinic Lerner College of Medicine: an innovative approach to medical education and the training of physician investigators. Acad Med 2007; 82: 390-6.

5. Altahawi F, Sisk B, Hicks C, Dannefer EF. Student perspectives on assessment: experience in a competency-based portfolio system. Med Teach 2012; 34: 221-5.

6. Cilliers FJ, Schuwirth LW, Adendorf HJ, Herman N, Van der Vleuten CP. The mechanism of impact of summative assessment on medical students' learning. Adv Health Sci Educ 2010; 15: 695-715.

7. Harden RM, Shumway JM. AMEE guide no. 25. The assessment of learning outcomes for the competent and reflective physician. Med Teach 2003; 25: 569-84.

8. Holmboe ES, Sherbino J, Long DM, Swing SR, Frank JR. The role of assessment in competency-based medical education. Med Teach 2010; 32: 676-82.

9. Schuwirth LWT, Van der Vleuten CP. Programmatic assessment: from assessment of learning to assessment for learning. Med Teach 2011; 33: 478-85.

10. Graber ML, Tompkins D, Holland JJ. Resources medical students use to derive a differential diagnosis. Med Teach 2009; 31: 522-7.

11. Elstein AS, Schwarz A. Clinical problem solving and diagnostic decision making: selective review of the cognitive literature. BMI 2002; 324: 729-32.

12. Norman G. Research in clinical reasoning: past history and current trends. Med Educ 2005; 39: 418-27.

13. Sandhu H, Carpenter C. Clinical decision making: opening the black box of cognitive reasoning. Ann Emerg Med 2006; 48: 713-9.

14. Gimeno-Sacristán J, Pérez-Gómez AI. Comprender y transformar la enseñanza. Madrid: Morata; 2002.

15. Artino AR, Holmboe ES, Durning SJ. Can achievement emotions be used to better understand motivation, learning, and performance in medical education? Med Teach 2012; 34: 240-4.

16. Pérez-Gómez A. Comprender y enseñar a comprender Reflexiones en torno al pensamiento de J. Elliot. In Elliot J. La investigación-acción en educación. Madrid: Morata; 1990.

17. Plaut SM, Baker D. Teacher-student relationships in medical education: boundary considerations. Med Teach 2011; 33: 828-33.

18. Dyrbye LN, Harper W, Durning SJ, Moutier C, Thomas MR, Massie FS, et al. Patterns of distress in US medical students. Med Teach 2011; 33: 834-9.

19. Hyden LC, Lumma A. Learning to talk and talking about talk: professional identity and communication technology. In Olin Lauritzen S, Hyden LC, eds. Medical technologies and the life world: the social construction of normality. New York: Routledge; 2007.

20. Lumma-Sellenthin A. Talking with patients and peers: medical students' difficulties with learning communication skills. Med Teach 2009; 31: 528-34.

21. Goethals RL, Darley JM. Social comparison theory: an attributional approach. In Suls J, Miller RL, eds. Social comparison processes: theoretical and empirical perspectives. Washington DC: Hemisphere; 1977.

22. Raat J, Kuks J, Cohen-Schotanus J. Learning in clinical practice: stimulating and discouraging response to social comparison. Med Teach 2010; 32: 899-904.

23. Blanton H, Buunk BP, Gibbons FX. When better-than-others compare upward: choice of comparison and comparative evaluation as independent predictors of academic performance. J Pers Soc Psychol 1999; 76: 420-30. 